\title{
Eye fixation and saccade during kana and kanji text reading: Comparison of English and Japanese text processing
}

\author{
NAOYUKI OSAKA \\ Kyoto University, Kyoto, Japan
}

\begin{abstract}
In Experiment 1, duration of eye fixations, saccade magnitude, saccade velocity, and regressive eye movements were measured during alphabet-like kana- (both katakana and hirakana) and logographic kanji-based Japanese text reading. The results suggest that katakana reading required longer fixation durations (about $240 \mathrm{msec}$ ) with small saccade magnitude (about $3^{\circ}$, which corresponds to 3.5 letters), whereas kanji-based reading required shorter fixation durations (about $170 \mathrm{msec}$ ) with larger saccade magnitude (about $5^{\circ}$, which corresponds to 6 letters). Eye movement characteristics of hirakana-based text fell in between. The frequency of the regressive eye movements was found to be significantly large for katakana text. In Experiment 2, interlanguage (English-Japanese) comparison was made with changing difficulty levels. The results showed that, for texts of the same difficulty, English required longer fixation durations from Japanese students than did Japanese texts, but no significant difference was found between difficulty levels. These results strongly suggest that the text processing for phonogram-based kana components and ideogram-based kanji components was different in terms of reading strategies, and that longer fixation durations were needed by Japanese students for reading English as compared with Japanese texts.
\end{abstract}

Eye movements have been measured during English text reading. Recent reviews on the role of eye movements during reading show that fixation duration would be a useful measure for the understanding of cognitive processing during text reading (Just \& Carpenter, 1987; McConkie \& Zola, 1987; Rayner \& McConkie, 1976; Rayner \& Pollatsek, 1987). It has been found that fixation duration changes from about 50 to $500 \mathrm{msec}$ as word frequency and difficulty level change within a phonogrambased orthography such as that of English. However, the eye movement characteristics appear different for an ideogram- (kanji-) based text such as Japanese (Osaka, 1987a). Normally, Japanese text is written in a kanji/kana mixed mode, but the same text can also be rewritten in either katakana or hirakana signs. Note that hirakana and katakana signs stand for the same sound-based phonetic symbols for syllables. There are two sets of 68 kana signs each, hirakana and katakana, which are functionally equivalent to one another, but their written signs are completely different in visual appearance. The major difference between kanji and kana would be found in their lexical access properties. The hypothesis here is that kanji and kana would have direct and indirect lexical access properties, respectively, and that the difference between them can be observed from eye movement behavior. The

This research was supported in part by Grants-in-Aid for Scientific Research (No. 62410003 and No. 63510055) from the Ministry of Education, Science and Culture. The author's address is: Department of Psychology, Faculty of Letters, Kyoto University, Kyoto 606, Japan. aim of the present study was to provide supporting evidence for this hypothesis, together with a comparison of English and Japanese in terms of eye movements.

\section{EXPERIMENT 1}

In Experiment 1, according to the hypothesis, eye movements were analyzed during katakana-, hirakana-, and kanji-based text reading.

\section{Method}

\section{Apparatus}

For eye movement recording, NAC's eye mark recorder system (Type V) was employed. The system can record the position of the right eye every $33 \mathrm{msec}$ with a sensor array device that is capable of receiving a very narrow infrared $(950 \mathrm{~nm})$ beam reflected from the cornea. Each eye position was recorded in a VTR system via a data output interfacing unit (V-99B type). The data were analyzed with a PC system (NEC's PC9801vx Model 2). An optional close-up lens providing a $30^{\circ}$ field, specially designed for recording eye movements during text reading, was used. A chinrest was used for exact recording.

\section{Materials}

Three different sets of a written Japanese text were used: katakana, hirakana, and kanji texts. The original text, selected from the work of a modern popular Japanese writer, was written in the normal kanji/hirakana mixed mode. The kanji contribution factor of this text was $\mathbf{0 . 4 0}$. This factor is the new index for evaluating the kanji contribution in a whole text; it is given by the following formula: kanji contribution factor $=$ (number of kanji characters)/(number of characters in whole text). Katakana and hirakana versions of the same text were also made. Each set had 320 characters and was presented on a monochrome CRT screen; each set, $20 \times 16$ characters, subtended a $17^{\circ} \times 20^{\circ}$ visual angle. A single character subtended a visual angle of $0.85^{\circ}$ from a $40-\mathrm{cm}$ viewing distance. 


\section{Procedure}

After calibration trials, each subject was asked to read the text silently as it appeared on the display screen. The subject was then asked to answer several questions regarding the story of the text after the session was over. The difficulty level for each text was rated on a 5-point scale $(1=$ easy, and $5=$ difficult $)$ by a different group of subjects beforehand.

The following measures taken from the 20-sec experimental sessions were analyzed (there were 600 frames for each condition):

Fixation duration. Fixation within the area of $1^{\circ}$ of spatial extent and longer than $66 \mathrm{msec}$ ( 2 frames) was selected from the fixation data.

Saccade magnitude. Saccade magnitude including the regressive saccadic movement within each line was collected, but the regressive movement due to return sweep (i.e., line change) was excluded.

Saccade velocity. Saccade velocity was measured as the ratio of the moved distance divided by the required time.

Regressive eye movements. Negative data along the $x$-axis were counted and their magnitudes calculated; but the return sweeps were excluded.

\section{Subjects}

Six college students enrolled in the psychology program were the subjects. Two students were allocated to each of the three different sets of the text. They had normal or corrected-to-normal right eye acuities.

\section{Results and Discussion}

Figure 1 shows the means and $1 S D$ units. Mean fixation durations across subjects were $238.91,190.78$, and 168.26 msec for katakana, hirakana, and kanji, respectively. The analysis of variance (ANOVA) revealed the significant main effect $[F(2,15)=8.80, p<.01]$. As Figure 1 indicates, kanji could decrease the fixation duration.

The mean saccade lengths were found to be $3.07^{\circ}$, $4.68^{\circ}$, and $5.01^{\circ}$ for katakana, hirakana, and kanji, respectively. The ANOVA revealed a significant main effect among these conditions $[F(2,15)=9.28, p<.01]$.
Kanji-based text requires a longer saccade than does kanabased text. It was found that the eye's landing points are likely to correspond to kanji components scattered within a hirakana context, with high probability. Thus, in kanjibased reading, kanji components play the role of stepping stones with respect to a series of the hirakana components (Osaka, 1987a). The size of the saccadic movement appears to be significantly small for katakana text as compared with kanji text.

Why can kanji be detected easily with short fixations during reading? One possible reason would be a category effect similar to the letter-digit phenomenon. A category effect would indicate that the ease with which a subject could recognize kana or kanji depends on what category the other items on the screen fall into (Jonides \& Gleitman, 1972). This implies that the subject could easily recognize kanji from hirakana, in spite of the saccadic suppression in the parafovea during saccade (Osaka, $1987 \mathrm{~b}$ ), before fully identifying the character itself due to kanji's complex physical features. The typical Japanese college student tends to read kanji-based text by using a strategy that involves parafoveal detection of kanji components while skimming through the hirakana syllables. As Figure 1 shows, mean saccade size for the kanji text was approximately $5.01^{\circ}$ (i.e., corresponding to 5.8 characters), and this value appears to agree with data reported by Kambe (1989), in which the average distance from fixation to fixation was found to be approximately 3 to 5 characters, using kanji-based text. Shorter fixation durations as well as large saccade magnitudes for kanji text suggest that kanji components facilitated the processing efficiency due to kanji's direct lexical access property as compared with kana. These data also support the find-
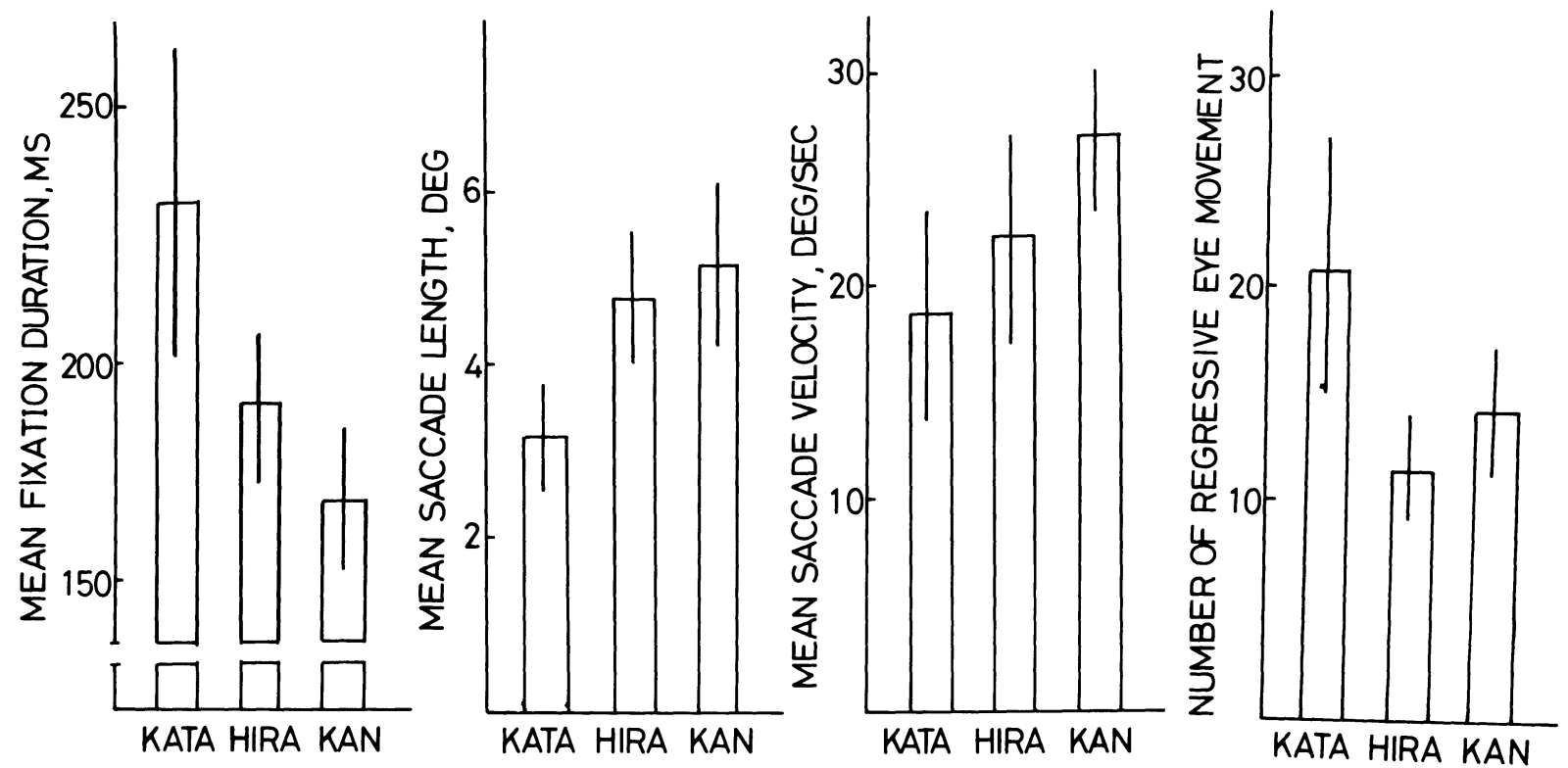

Figure 1. Mean fixation duration (in msec), mean saccade length (in deg), mean saccade velocity (in deg/sec), and number of regressive eye movements, as a function of text presentation mode. KATA, HIRA, and KAN indicate katakana, hirakana, and kanji, respectively. The vertical bar shows standard deviation. 
ing that a reader could change reading strategies by controlling the saccade size (O'Regan, 1980) in order to optimize the processing efficiency.

Means for saccadic velocity (note that the unit is not wpm) were $18.26^{\circ}, 21.94^{\circ}$, and $26.62^{\circ} / \mathrm{sec}$ for katakana, hirakana, and kanji, respectively, and the ANOVA indicated the significant main effect differences $[F(2,15)=$ $3.74, p<.05]$. Again kanji appears to facilitate processing efficiency.

The numbers of regressive eye movements were again found to be statistically significant $[F(2,15)=5.08, p<$ $.05]$, but the magnitude of regressive movement was not statistically significant.

The rated difficulty was 3.6, 3.0, and 2.5 for katakana, hirakana, and kanji, respectively. The fixation duration data appear to support this; as rated reading difficulty increased, fixation duration increased.

Most of the coefficients of variation tended to remain within the range between 40 and 130 for each text.

\section{EXPERIMENT 2}

In Experiment 2, English texts were introduced for comparison with Japanese text processing. In addition, difficulty levels regarding text content were controlled.

\section{Method}

\section{Apparatus}

The apparatus was the same as in Experiment 1.

\section{Materials}

Four sets of texts were employed: easy and difficult versions of both the English and the Japanese texts. One of the English texts was a trans-

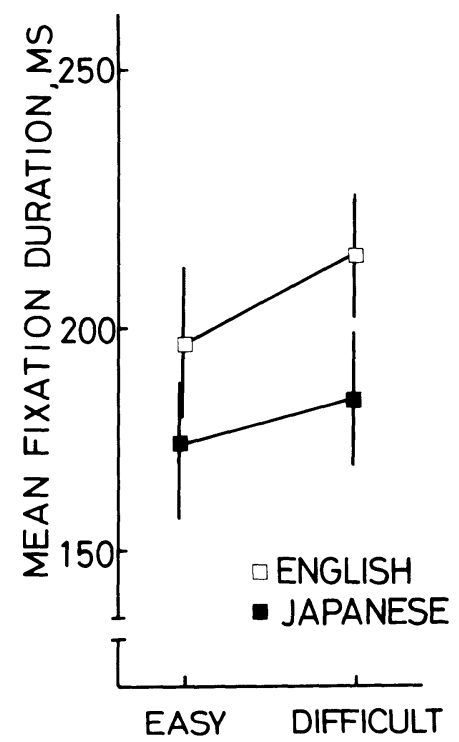

Figure 2. Mean fixation duration (in msec) as a function of difficulty of the text. The open and filled squares indicate the English and Japanese texts, respectively. lated version of the popular novel text (easy level; rated 2.4) used in Experiment 1, and the other was taken from a political article that appeared in New Yorker magazine (difficult level; rated 4.5). One of the Japanese texts was from a fairy tale for children (easy level, with a kanji contribution factor of 0.33 ; rated 2.0), and the other was from a philosophical essay (difficult level, with a kanji contribution factor of 0.53 ; rated 4.3). Both Japanese texts were written in the kanji-based mode.

\section{Procedure}

Fixation duration, saccade magnitude, saccade velocity, frequency of regressive eye movements, and magnitude of regressive eye movements were measured. The design was a 2 (language) $\times 2$ (difficulty) entry model.

\section{Subjects}

Four college students enrolled in the psychology program served as subjects. Each subject participated in four sets of text-reading sessions. They had normal or corrected-to-normal right eye visual acuities.

\section{Results and Discussion}

The ANOVAs for fixation duration, saccade length, velocity, and regressive movements revealed no significant main effects differences except for the main effect on language difference for fixation duration $[F(1,12)=5.84$, $p<.05]$. Figure 2 shows the results for this fixation duration condition. For the average Japanese reader, the English text appears to require longer fixation durations for both easy (195.00) and difficult (216.50) texts. However, the main effect of difficulty did not reach a statistically significant level $(p<.10)$, in spite of the difference in rated difficulty levels.

\section{REFERENCES}

Jonides, J., \& Gleitman, H. (1972). A conceptual category effect in visual search: $\mathrm{O}$ as letter or as digit. Perception \& Psychophysics, $12,457-460$

JUST, M. A., \& CARPENTER, P. A. (1987). The psychology of reading and language comprehension. Boston: Allyn \& Bacon.

KAMBE, N. (1989). The span of the effective visual field during a fixation in reading eye movement. Research Reports of the National Japanese Language Institute, 10, 59-80. (In Japanese, with English summary)

McConkIE, G. W., \& ZoLA, D. (1987). Visual attention during eye fixations while reading. In M. Coltheart (Ed.), Attention and performance XII: The psychology of reading (pp. 385-401). Hillsdale, NJ: Erlbaum.

O'Regan, J. K. (1980). The control of saccade size and fixation duration in reading: The limits of linguistic control. Perception \& Psychophysics, 28, 112-117.

OSAKA, N. (1987a). Effect of peripheral visual field size upon eye movements during Japanese text processing. In J. K. O'Regan \& A. LevySchoen (Eds.), Eye movements: From physiology to cognition (pp. 421429). Amsterdam: Elsevier.

OSAKA, N. (1987b). Variation of saccadic suppression with target eccentricity. Ophthalmic \& Physiological Optics, 7, 499-501.

RAYNER, K., \& MCConKIE, G. W. (1976). What guides a reader's eye movements? Vision Research, 16, 829-837.

Rayner, K. J., \& Pollatsek, A. (1987). Eye movements in reading: A tutorial review. In M. Coltheart (Ed.), Attention and performance XII: The psychology of reading (pp. 327-362). Hillsdale, NJ: Erlbaum.

(Manuscript received April 10, 1989.) 